\title{
STAX: a new technique for detecting Axions
}

\author{
Paolo Spagnolo* \\ INFN Pisa \\ E-mail: Paolo.spagnolo@cern.ch
}

\begin{abstract}
An improved detection scheme for a light-shining-through-wall (LSW) experiment for axion-like particle searches is discussed. In this proposal it is suggested the use of gyrotrons as source of photons, which can provide extremely intense fluxes at frequencies around $30 \mathrm{GHz}$; transitionedge-sensors (TES) single photon detectors in this frequency domain, with efficiency $\approx 1$; high quality factor Fabry-Perot cavities in the microwave domain, both on the photon-axion conversion and photon regeneration sides. With this set-up, current laboratory exclusion limits on axion-like particles might be improved by at least four orders of magnitude for axion masses $\lesssim 0.02 \mathrm{meV}$.
\end{abstract}

38th International Conference on High Energy Physics 3-10 August 2016

Chicago, USA

${ }^{*}$ Speaker. 


\section{Introduction}

Axions [1] are among the most popular dark matter candidates. They are light neutral scalar or pseudoscalar bosons, with mass $m_{a} \approx \mu \mathrm{eV}-m \mathrm{eV}$, coupled to the electromagnetic field via

$$
\mathscr{L}_{I}=\frac{1}{4} G a F^{\mu v} \tilde{F}_{\mu v}
$$

In QCD axion models (DFSZ [2] and KSVZ [3]), the axion-photon coupling constant $G$ is directly related to $m_{a}$; thus, $G$ is the only free parameter of the theory. In axion-like particle (ALP) searches, the parameter space is extended: $G$ and $m_{a}$ are the free parameters [4].

Axions and ALPs experimental searches can be divided into two main categories: 1) Axions from stellar and space sources; 2) Laboratory searches. In the former case, exclusion limits on the axion-photon coupling constant are provided by estimates of stellar-energy losses [4, 5], helioscope $[6,7,8]$ and haloscopes experiments[6,9]. In the latter case, limits on $G$ are given by photon polarization [10] and Light-Shining-Through-Wall (LSW) [6, 11, 12, 13, 15] experiments.

This proposal is an improved version of the LSW experiments, achieved with the use of high luminosity source of photons, obtained moving to the sub- $\mathrm{THz}$ region where high luminosity sources of photons, like gyrotrons or klystroms can operate, thanks to a cross-section of the Primakoff photon-axion interaction that is almost independent on the photon energy in the sub-eV region, where the search is performed. The implemented solution would improve of the present ALPs laboratory limits on $G$ by at least four orders of magnitude [16], by using extremely intense photon fluxes from gyrotron sources at frequencies around $30 \mathrm{GHz}$, TES single photon detectors with efficiency $\approx 1$, and high quality factor Fabry-Perot cavities in the microwave domain $\left(Q \approx 10^{4}-10^{5}\right)$, both on the photon-axion conversion and photon regeneration sides.

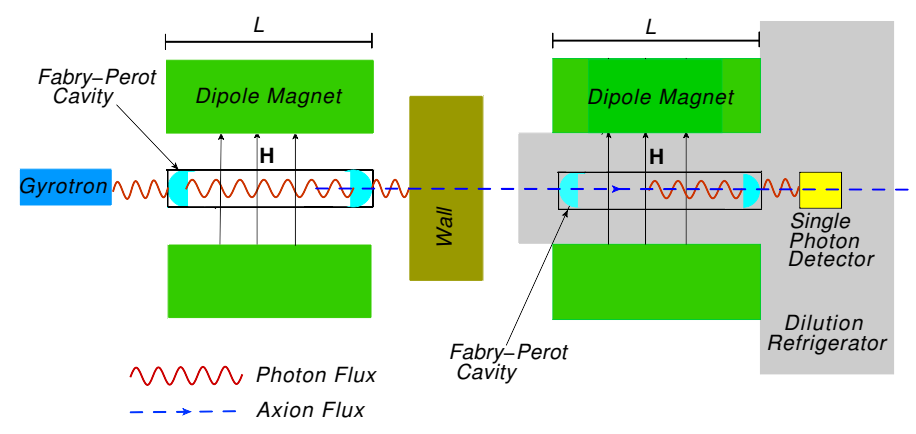

Figure 1: Experimental configuration of the STAX LSW experiment.

\section{LSW experiments}

A typical LSW experiment is given by two intense magnetic fields $\mathbf{H}$, separated by a thick wall $[6,11,12,13,15]$. Axions can be produced in the magnetic field before the wall, from a source of photons via Primakoff effect. Photons exchange 3-momentum q with $\mathbf{H}$, but the energy is conserved.

The photons which do not convert into axions are stopped by an optical barrier, "the wall", while axions can cross the wall, due to their negligible cross-section with ordinary matter. On the 
other side of the wall in the second magnetic field, axions can back-convert to photons. Reconverted photons are detected via a single-photon detector.

The photon-axion-photon rate is given by

$$
\frac{\mathrm{d} N_{\gamma}}{\mathrm{d} t}=\Phi_{\gamma} \eta P_{\gamma \rightarrow a} \times P_{a \rightarrow \gamma} \sim \Phi_{\gamma} \eta G^{4} H^{4} L^{4}
$$

where $\Phi_{\gamma}\left[\mathrm{s}^{-1}\right]$ is the initial photon flux and $\eta$ the single-photon-detector efficiency, $\mathrm{G}$ is the (unknown) photon-axion coupling and $\mathrm{L}$ is the length of the photons path in the magnetic field $\mathrm{H}$. $P_{\gamma \rightarrow a}=P_{a \rightarrow \gamma}$ is the axion-photon conversion probability. Since $\mathrm{G}$ is $\leq 10^{-10}$ from current experimental constraints, the probability of such a double conversion is extremely low due to this 4 th power of G. Nevertheless LSW experiments have the advantage to be independent on cosmological prediction and to exactly know the energy and the time of the searched axions. The rate can be increased by introducing a Fabry-Perot cavity in the magnetic field area before the wall by a factor of $Q$, which is the quality factor of the cavity. Moreover, as discussed in Ref. [17], the rate can be further increased with the addition of a second Fabry-Perot cavity in the magnetic field region beyond the wall. In Fig. 1 the scheme of our proposal is sketched.

\section{STAX experimental configuration and calculated exclusion limits}

Current experimental limits for the axion-photon coupling constant are given by the ALPS Collaboration [11]. The upgrade ALPS-II [13], will improve the previous limits mainly by increasing the magnetic field length as well as introducing a second cavity in the magnetic field region behind the wall. ALPS-II configuration is very similar to that of Fig. 1, with the photon flux provided by an optical laser. The STAX proposal consists in a new generation LSW experiment and improve the limits on $G$ by using sub-THz photon sources, like gyrotrons, providing very high luminosity (up to $10^{28}$ photons/s) at frequencies of about $30 \mathrm{GHz}$, resulting in photon fluxes up to $10^{10}$ more intense than those from optical lasers, used in previous LSW experiments.

The number of expected events will grow by a factor of Q if a Fabry-Perot resonant cavity (for $30 \mathrm{GHz}$ photons) with quality factor $\mathrm{Q}$ encloses the magnetic field region where photon-to-axion conversion is expected to occur. Standard quality factors of Fabry- Perot cavities in the microwave domain are know to be $Q=10^{4} \div 10^{5}$.

Single-photon detectors for light at these frequencies, with almost zero dark count can be developed, based on the (Transition-Edge-Sensor) TES technology. Calorimeters based on TES have been successfully exploited as single-photon quantum detectors for X-ray spectroscopy[14] as well as for secure quantum communication applications using near-IR photons. Nano-calorimeters are able to extend the ability of conventional calorimeters to detect both very tiny powers as well as small amounts of energy which potentially correspond to a single sub-THz or microwave photon event. The TES detector will be coupled to an antenna and operated at ultra-cold temperatures $\approx 10$ $\mathrm{mK}$, to achieve an almost negligible background with an efficiency close to 1 . Thanks to such a device, the present laboratory exclusion limits on axion-like particles might be improved by at least four orders of magnitude for axion masses $\lesssim 0.02 \mathrm{meV}$ [16]. Liimits that STAX experiment may reach are compared to previous experimental results in Fig. 2. For this proposal we have assumed a intense dipole magnetic field $\mathrm{H}=15 \mathrm{~T}$ with a path $\mathrm{L}=50 \mathrm{~cm}$, an exposure of one month and zero dark counts. STAX and STAX 2 use gyrotrons with power of $100 \mathrm{~kW}$ and $1 \mathrm{MW}$ respectively. 


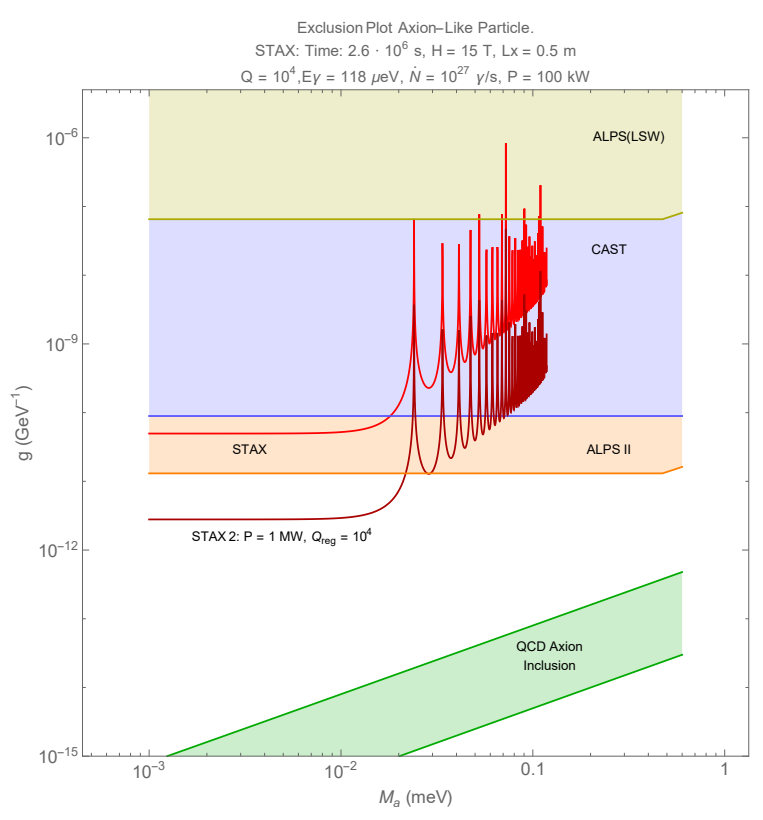

Figure 2: 90\% CL STAX and STAX 2 expected limits on G in case of a null result for axions with $m_{a} \lesssim$ $0.02 \mathrm{meV}$. An exposure time of one month and zero dark counts are considered. "STAX" and "STAX 2" configurations correspond to a $100 \mathrm{~kW}$ and $1 \mathrm{MW}$ gyrotron sources, respectively.

\section{References}

[1] R.D. Peccei and H. Quinn, "CP Conservation in the Presence of Instantons," Phys. Rev. Lett. 38, 1440 (1977); S. Weinberg, “A New Light Boson?," Phys. Rev. Lett. 40, 223 (1978); F. Wilczek, "Problem of Strong p and t Invariance in the Presence of Instantons," Phys. Rev. Lett. 40, 279 (1978).

[2] M. Dine, W. Fischler, and M. Srednicki, "A Simple Solution to the Strong CP Problem with a Harmless Axion," Phys. Lett. B 104, 199 (1981); A.R. Zhitnitsky, "On Possible Suppression of the Axion Hadron Interactions," Sov. J. Nucl. Phys. 31, 260 (1980).

[3] J.E. Kim, "Weak Interaction Singlet and Strong CP Invariance," Phys. Rev. Lett. 43, 103 (1979); M.A. Shifman, A.I. Vainstein, and V.I. Zakharov, "Can Confinement Ensure Natural CP Invariance of Strong Interactions?," Nucl. Phys. B 166, 493 (1980).

[4] See the review "Axions and Other Similar Particles" on K. A. Olive et al. [Particle Data Group Collaboration], "Review of Particle Physics," Chin. Phys. C 38, 090001 (2014).

[5] M.S. Turner, "Windows on the Axion," Phys. Rep. 197, 67 (1990); G.G. Raffelt, "Astrophysical methods to constrain axions and other novel particle phenomena," Phys. Rep. 198, 1 (1990).

[6] P. Sikivie, “Experimental Tests of the 'Invisible' Axion,” Phys. Rev. Lett. 51, 1415 (1983).

[7] M. Arik et al. [CAST Collaboration], "New solar axion search using the CERN Axion Solar Telescope with ${ }^{4}$ He filling,” Phys. Rev. D 92 (2015) 2, 021101.

[8] E. Armengaud et al. [IAXO Collaboration], "Conceptual Design of the International Axion Observatory (IAXO)," JINST 9, T05002 (2014); T. Dafni et al. [IAXO and CAST Collaborations], “Axion helioscopes update: the status of CAST \& IAXO," PoS TIPP 2014, 130 (2014).

[9] S. J. Asztalos et al. [ADMX Collaboration], "A SQUID-based microwave cavity search for dark-matter axions," Phys. Rev. Lett. 104, 041301 (2010). 
[10] L. Maiani et al., "Effects of Nearly Massless, Spin Zero Particles on Light Propagation in a Magnetic Field," Phys. Lett. B 175, 359 (1986); Y. Semertzidis et al., "Limits on the Production of Light Scalar and Pseudoscalar Particles," Phys. Rev. Lett. 64, 2988 (1990); E. Zavattini et al. [PVLAS Collab.], "Experimental observation of optical rotation generated in vacuum by a magnetic field," Phys. Rev. Lett. 96, 110406 (2006); E. Zavattini et al. [PVLAS Collab.], "New PVLAS results and limits on magnetically induced optical rotation and ellipticity in vacuum," Phys. Rev. D 77, 032006 (2008).

[11] K. Ehret et al. [ALPS Collaboration], "New ALPS Results on Hidden-Sector Lightweights," Phys. Lett. B 689, 149 (2010).

[12] M. Betz, F. Caspers, M. Gasior, M. Thumm and S. W. Rieger [CROWS Collaboration], "First results of the CERN Resonant Weakly Interacting sub-eV Particle Search (CROWS),” Phys. Rev. D 88, no. 7, 075014 (2013).

[13] R. Bähre et al. [ALPS-II Collaboration], "Any light particle search II. Technical Design Report," JINST 8, T09001 (2013); B. Döbrich [ALPS-II Collaboration], "What's new in ALPS-II," arXiv:1309.3965.

[14] K.D. Irwin, G.C. Hilton, Cryogenic Particle Detection, Vol. 99, Springer-Verlag, Berlin, 2005, p. 63.

[15] R. Ballou et al. [OSQAR Collaboration], "New Exclusion Limits for the Search of Scalar and Pseudoscalar Axion-Like Particles from "Light Shining Through a Wall", arXiv:1506.08082.

[16] L.M. Capparelli, G. Cavoto, J. Ferretti, F. Giazotto, A.D. Polosa and P. Spagnolo, "Axion-like particle searches with sub-THz photons," Phys. Dark Univ. 12, 37 (2016).

[17] P. Sikivie, D.B. Tanner, K. van Bibber, "Resonantly enhanced axion-photon regeneration," Phys. Rev. Lett. 98, 172002 (2007). 